\title{
Tree based MultiPath Data Diffusion for Multimedia Wireless Sensor Networks
}

\author{
Muhammad Zaid Hameed, Arslan Shahid, Saad Ahmad Khan and Zubair Ahmad Khan \\ Department of Electrical Engineering \\ University of Engineering \& Technology, Lahore Pakistan \\ Email: \{mzaidhameed, ars.shahid\}@gmail.com, \{saad.ahmad, zubair.khan\}@uet.edu.pk
}

\begin{abstract}
Wireless Multimedia Sensor Networks (WMSNs) have come forth as a significant technology with striking performance in processing of multimedia signals and increase an encompassing range of real time applications. However, WMSNs have strict restraints with respect to available resources in terms of bandwidth, energy and processing capacities of sensor nodes. So, in place of a stand alone video sensor node, it is suitable to use multiple video sensors which operate the acquired information locally and intercommunicate with each other to draw out the crucial data and send it to the sink. Thus, in-network collaborative processing renders solutions to multimedia applications with decreased processing power, data delivery response time and energy consumption. In this paper, we present a novel multipath construction scheme which builds disjoint, minimum hop and energy efficient multiple paths between base station and video sensor nodes and between different video sensor nodes for in-network collaborative processing. On demand path construction priorities can be assigned according to the situation. Performance evaluation of our suggested path construction scheme demonstrates its effectivity for collaborative video sensor networks.
\end{abstract}

Index Terms-Wireless Sensor Networks, Disjoint multi-path, Collaborative Wireless Multimedia Sensor Networks.

\section{INTRODUCTION}

Recent growth of multimedia (image and audio) sensor technology has provided the low-cost, low-power multimedia hardware such as CMOS cameras and microphones functional for small sensor nodes for heightened event description and thus facilitated in the assembling of wireless multimedia sensor networks (WMSN). Consequently, WMSNs have emerged as an important technology with outstanding performance in multimedia signal processing and increase a broad range of applications such as home area networking [1], battlefield surveillance networks, advanced health monitoring systems, target detection and tracking, disaster relief operations and industrial process control [2] [3] [4].

Wireless multimedia sensor networks have bounded energy supply, bandwidth and processing capacities, whereas most of multimedia applications demand intensive computational processing which leads to increased energy consumption and delays in data delivery. So, in place of a stand-alone video sensor node, it is suitable to use a number of video sensor nodes which operate the acquired information locally, communicate with each other to share the information and send only critical data to the base station. So, the total amount of data sent is reduced which leads to reduction of latency in data delivery [5]. Thus, in-network collaborative processing renders a solution with decreased processing power, energy consumption and data delivery latency for multimedia applications. Task mapping and scheduling is performed for innetwork cooperative processing [6] [7]. Such communication necessitates the construction of paths between base station and videos sensors and between video sensors themselves for a network with bandwidth limitations. In order to overcome the bandwidth restraints of multimedia content transmission, multipath routing is requisite because a single path is unable to accomplish the high bandwidth demand. Due to timeliness requisites, only those paths from all discovered multipaths are suitable which satisfy some end-to-end delay restraints in transmission of data.

The underlying features of WMSNs have guided the researchers towards the development of multipath routing strategies to establish multiple paths between source and sink. Some multipath routing approaches center on reliability [8], timeliness in delivery of data and increase in effective data rate [9] [10] [11]. Most of these techniques are optimal with respect to a single parameter i.e. these approaches either minimize energy consumption or hop delays but not both simultaneously, thus are less desirable for some practical applications. Furthermore, these strategies are established for a single video sensor node, therefore, unsuitable for in-network collaboration.

In this paper we present a novel strategy for construction of multiple paths which minimizes end-to-end delay in an energy efficient way. This multipath construction algorithm finds its cornerstones in Rapidly-Exploring Random Trees, a renowned path planning technique in robotics, computer graphics and virtual prototyping [12]. Our proposed scheme builds multiple paths between video sensor nodes themselves and with sink (base station) by formation of trees which quickly develop in a randomized manner through the network and pick out paths on the basis of minimum end-to-end delays and energy consumption. Performance evaluation of our suggested scheme is carried out in different network configurations. Our algorithm outperforms the different multiple path construction approaches [8] [13] and simulation results show its effectivity for collaborative video sensor networks.

The rest of the paper is organized as follows. Section II II imparts a summed up discussion of related work. Section 
III defines our system model. We present our multipath construction algorithm in Section IV. Simulation of our proposed algorithm and results are presented in Section V. In the end, we conclude our paper in Section VI.

\section{RELATED WORK}

In this paper [14], a multipath construction technique based on geographic routing: TPGF is proposed. TPGF discovers maximum node-disjoint energy optimal paths with minimal path length and end-to-end delays. The algorithm completes in two levels in which it tries to discover potential paths and then makes optimal paths with least number of hops. MPMPS [9], a multipath selection strategy with multiple priorities is proposed in transport layer. This approach provides multiple priorities for multimedia content on the basis of end-toend transmission delays and contextual information. MPMPS picks out maximum node-disjoint paths to maximize the transmission throughput of multimedia content by warranting end-to-end transmission delays. In this paper [15], a path formation technique is proposed with incremental multipath construction approach which establishes a single path or on demand multiple paths in case of path congestion or bandwidth limitations. Each sensor node keeps up and brings up to date a path table which records the different paths to the sink based on path id, a parametric quantity for path quality and next hop neighbour toward the sink for this path.

In this paper [16], an altered version of hierarchical clustering algorithm LEACH has been developed for the establishment of multiple paths between the elected cluster heads. As a result, constructed multiple paths enhance the available transmission rate and two presented scheduling algorithms enhance the execution in terms of power consumption and perceived video quality.

MMSPEED [10] formulates a geographic routing technique which furnishes probabilistic quality of service assurance in reliability and timeliness fields by allowing for multiple speed selections with adaptive modifications according to delay limitations. Reliability is attained by proposed multipath routing which is dependent upon end-to-end transmission probability. These QOS assurances are accomplished by localized decisions without global network information.

\section{NETWORK MODEL}

In this paper, we consider a heterogeneous wireless multimedia sensor network which consists of a base station, video sensor nodes and a large number of scalar sensor nodes. All of the sensor nodes are randomly deployed and they remain at their locations after the deployment. The sensor nodes under consideration do not require the information of their geographical coordinates for their routing decisions thus they are not equipped with any position estimating device e.g. GPS (Global Positioning System). A base station or the sink node is the most resourceful element in terms of higher energy resources, intensive computational capabilities and large data rates hence it is considered to be a personal computer. Video sensor Nodes have to perform energy demanding multimedia sensing

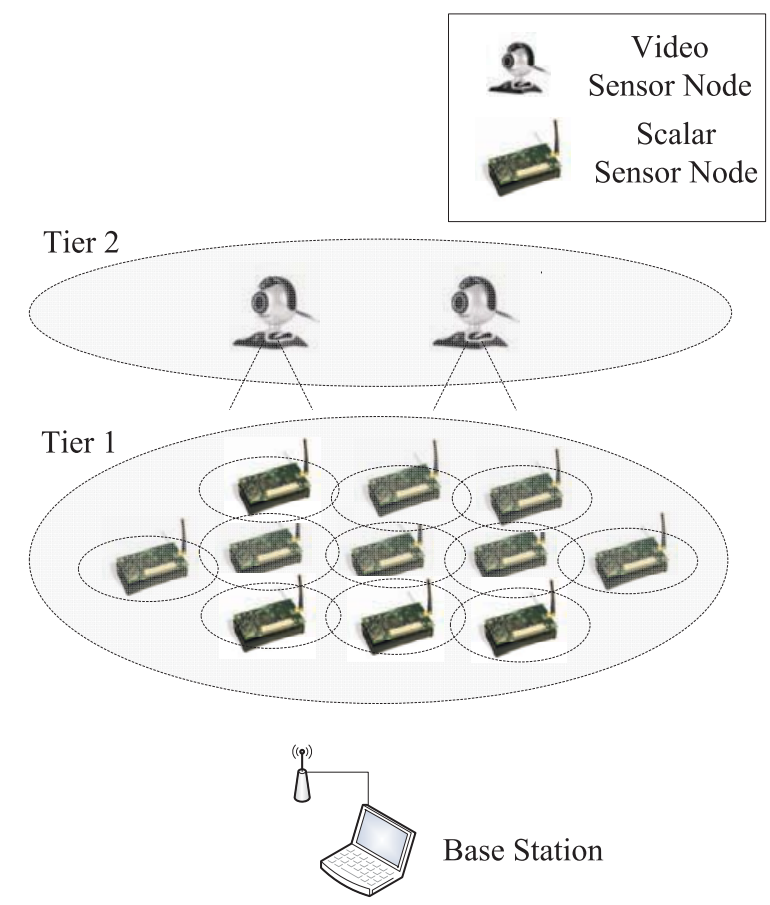

Fig. 1. Network architecture for Wireless Multimedia Sensor Network

operations which also require high computational power, so these video sensor nodes are equipped with large battery resources, processor and wireless transceiver with high data rates. Scalar sensor nodes are used for scalar sensing and for routing multimedia content from video node to base station. It is also assumed that all sensor nodes can dynamically adjust their transmission radius by adjusting their transmission power according to requirement.

This heterogeneous network of sensor nodes under consideration is a two-tier network as shown in Figure 1, which supports for scalar sensing measurements (Tier 1) and for multimedia content (image, video and audio streams) measurement (Tier 2). The fundamental design principle is to present each task requested by the application to the lowest tier with sufficient resources to execute the task [2]. Sensor nodes from higher tiers are in sleep mode and waken only ondemand. So, this type of system saves a considerable amount of energy with a comparable performance in surveillance as compared to a single-tier system. Furthermore, multiple video sensor nodes operating simultaneously can process information collaboratively and send only critical data back to base station. Our focus is on developing a multipath construction algorithm to work in such a collaborative network of video sensor nodes. In this type of network video sensor nodes can share their multimedia information with each other through multiple paths for collaborative processing and then send the processed data through multiple paths to base station. These multipaths are formed by construction of trees which rapidly grow in a randomized manner through the network and select 
disjoint multiple paths based on minimum end-to-end delays and energy dissipation.

\section{Tree Based Disjoint Multi-Path Construction ALGORITHM}

We present a multipath construction algorithm that grows random trees, one of them rooted at the base station and other are rooted at each of the video sensor node (VSN) respectively. These trees are constructed to find

- Multiple paths between base station and different video sensor nodes.

- Multiple paths between different video sensor nodes for collaborative processing

Initially a network of $N$ senor nodes is created. A small number of sensor nodes $M$ have the capabilities for multimedia sensing and the rest of them $N-M=K$ sensor nodes are for scalar sensing. When the most recent nodes added to branches of different random trees are in the sensing radius of each other, a link is established between them and we say that a path is constructed.

Now we demonstrate the algorithm in detail.

\section{Initialization Of Trees:}

To begin algorithm, we define a random tree $\tau_{B S}$ for the base station and a set of random trees $T$ for a set $V$ of video sensor nodes (VSN) for a total number of video sensor nodes $V S N_{\text {count }}$ where,

$$
\begin{aligned}
& V=\sum V_{i} \mid V \subset V S N \\
& V_{i} \in V S N: i>0 \wedge i<V S N_{\text {count }} \\
& T=\left\{\tau_{V S N} \mid \tau_{V S N} \text { is a tree rooted at a } V S N \in V\right\}
\end{aligned}
$$

Each of these random trees is initialized to hold a single video sensor node. Tree $\tau_{B S}$ is initialized to hold the base station. Base station and all active video sensor nodes broadcasts a message containing an association request and a parameter which denotes their corresponding hop distances for the other sensor nodes in their sensing radius respectively. Nodes in the coverage area of each of them get associated by unicasting a message back to their corresponding query generating source nodes and update their hop distance attribute according to the association request message. All of the recently discovered nodes are the first hop neighbors of the corresponding source nodes, we will call them as first level child nodes.

\section{Minimum Distance Branch Formation:}

Next, each one of the recently discovered nodes of the different trees will be searched and minimum distance nodes in turn, become as the source node. These source nodes will send an association request for the other undiscovered sensor nodes in the network in the same fashion as described above. This minimum distance criterion is adopted for energy conservation and can be estimated by some metric according to application and depends on the hardware specifications of the nodes e.g. Received Signal Strength Indicator (RSSI). So, the nodes

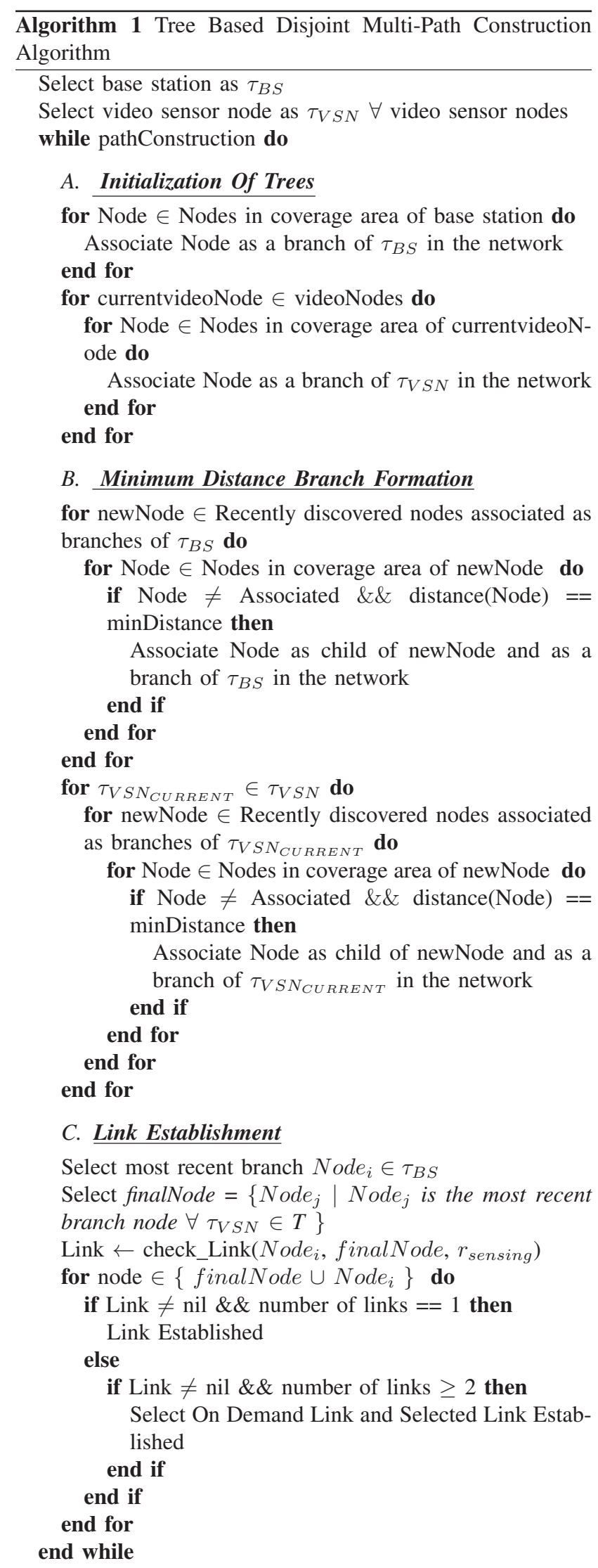




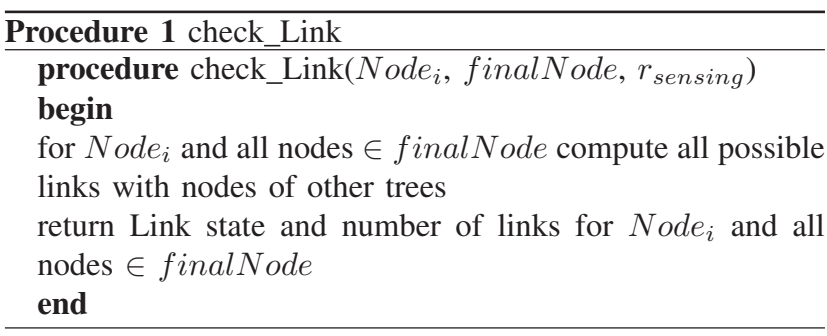

which are not associated yet and lie at minimum distance will be selected and associated as the next branch of the corresponding trees.

\section{Link Establishment:}

In this step, all of the most recently discovered nodes or higher level child nodes of different trees will send a request to associate with each other. If these nodes are in the sensing radius of each other they will get linked by a unicast message containing linking request. After that, a link is established by unicasting a message containing link established information to their corresponding parent nodes in the trees until they reach the corresponding base station or video sensor node. In the case, when these nodes are not in the sensing radius of each other, they will repeat the phase IV-B of the algorithm until the whole network is explored. There might be a case when nodes of more then two trees are in the sensing radius of one another. In such a situation, on demand priorities can be assigned to establish a link either to base station or to the video sensor node according to situation. Entire multipath construction scheme is shown in Algorithm 1.

The algorithm grows random trees which rapidly explore the entire network and thus construct multiple disjoint (non overlapping) paths which are of minimum hop delays and energy dissipation. This is ensured by selecting nearest next hop sensor node as the next branch of the tree. On demand priorities can be assigned to establish a link either to a base station or to a video sensor node according to situation which make this algorithm flexible for collaborative processing of multimedia content and its transmission to the base station.

\section{Simulations And Results}

In order to demonstrate our proposed multipath construction algorithm, we have used MATLAB for simulation of the algorithm.

\section{A. Simulation Model}

The simulations have been carried out for a heterogeneous wireless multimedia sensor network for battlefield surveillance which consists of video sensor nodes (VSN), scalar sensor nodes ( $\mathrm{SSN}$ ) and a base station (BS). We also assume that all of these sensor nodes (VSN, SSN and BS) once they have been deployed, remain at their location. For simulation purpose, a uniformly deployed sensor network of 100 sensor nodes in an area of dimensions $100 \mathrm{~m} \times 100 \mathrm{~m}$ is considered. Each sensor node has a sensing radius $r_{\text {sensing }}=40 \mathrm{~m}$. Base station is situated at one side of network at coordinates $(0,50)$ and two video sensor nodes are situated at the other side of network at coordinates $(100,0)$ and $(100,100)$ respectively. For the presented simulation scenario, Figure 2 shows finalized constructed paths just before link establishment and finalized paths explored after link establishment are shown in Figure 3.

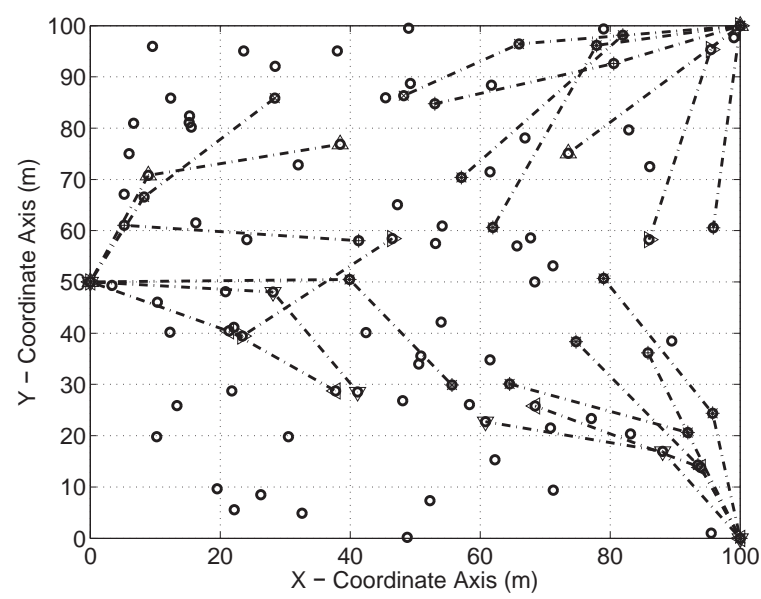

Fig. 2. Finalized paths before link establishment for two dimensional Uniform Deployment

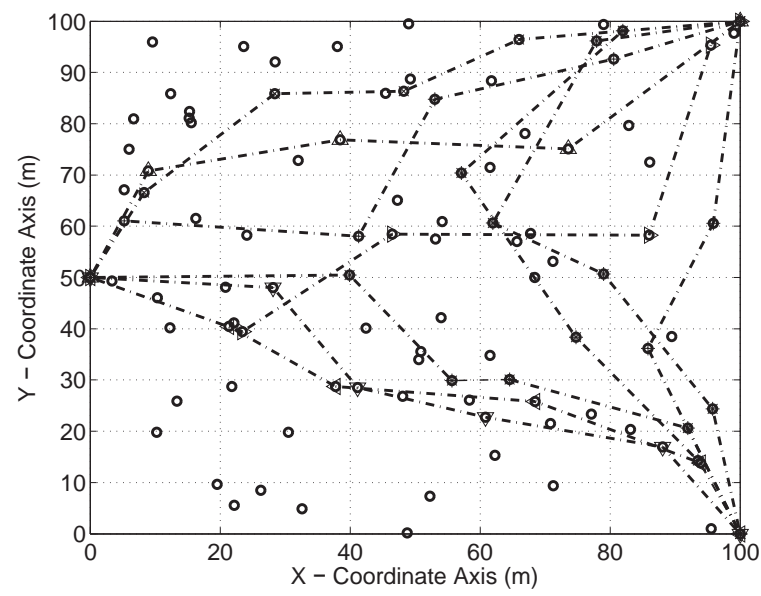

Fig. 3. Finalized paths after link establishment for two dimensional Uniform Deployment

To prove the effectiveness of our algorithm, we have compared it with another disjoint multipath formation scheme discussed in [13]. This algorithm [13], constructs on-demand multiple disjoint paths using request/reply messages. Initially, base station has no information known, it floods the ROUTE REQUEST (RREQ) message containing the source ID and sequence number to the entire network. Intermediate nodes add their IDs and forward the packet. Through different routes, several copies of the message reach the destination node which in turn selects multiple disjoint paths and sends ROUTE REPLY (RREP) packets back to the base station via the selected paths. These selected paths are not of equal 
lengths. We have simulated both algorithms for various network topologies formed by deployment of altering number of sensor nodes (each of sensing radius $r_{\text {sensing }}=40 \mathrm{~m}$ ) in a network of area $100 \mathrm{~m} \times 100 \mathrm{~m}$. In order to reduce the effect of a specific network configuration, simulation for each uniformly deployed network of varied number of sensor nodes is carried out 10 times. In Figure 4, averaged total number of disjoint paths explored (between two video sensor nodes and the base station) are presented for such topologies. It can be seen that our proposed scheme has more disjoint paths between the base station and video sensor nodes which is of particular interest in WMSNs. Through Increased number of multiple paths, network lifetime increases as we can shuffle between paths for transmission.

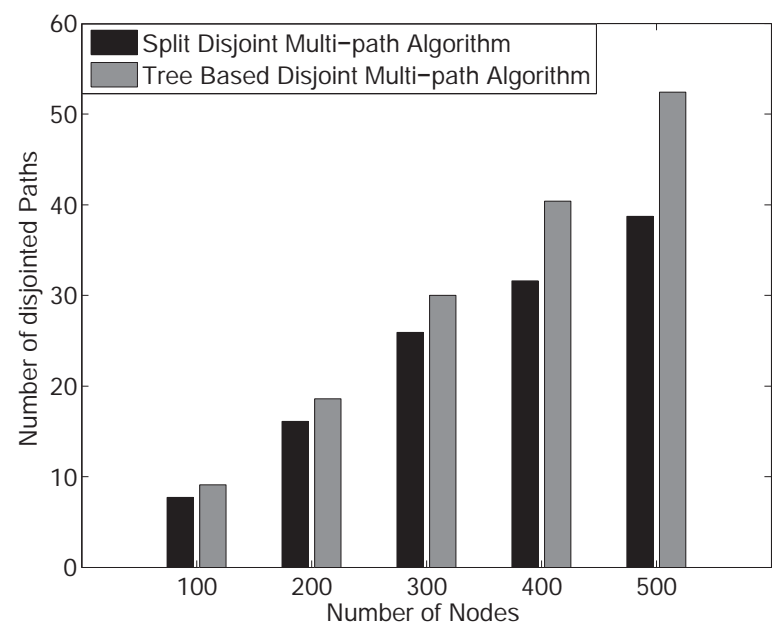

Fig. 4. Total number of paths explored between video sensor nodes and base station for two dimensional Uniform Deployment in a network of dimensions $100 \mathrm{~m} \times 100 \mathrm{~m}$

\section{B. Energy Model}

We consider an energy model [17] which assumes that for a sensor node, energy consumption is prevailed by wireless transmissions and receptions while omitting the energy consumption in sensing and computations performed at the nodes. This assumption is rationalized by the fact that the consumption of energy for wireless transmissions and receptions is much greater then the energy consumed for processing and sensing.

We apply the first order radio model as shown in Figure 5, which assumes energy loss with respect to square of distance for channel transmissions. So, if a transmission of a $k$-bit data packet is required to a node which is at a distance $r$, the energy consumed in the radio module will be $E_{T x}(k, r)$ where

$$
E_{T x}(k, r)=E_{E l e c} \times k+E_{a m p} \times k \times r^{2}
$$

and for the receiver, energy consumed to receive this $k$-bit data packet will be given by $E_{R x}(k)$ as follows,

$$
E_{R x}(k)=E_{E l e c} \times k
$$

where $E_{\text {Elec }}$ (Joule/bit) is the energy consumed to excite the transmitter or receiver circuitry and $E_{a m p}\left(\mathrm{Joule} / \mathrm{bit} / \mathrm{m}^{-2}\right)$ is the energy consumed for the transmitter amplifier circuit to transmit at a distance ' $r$ '. From the equations 1 and 2, it can be seen that energy consumed for transmission and reception is directly proportional to number of bits in the data packet.

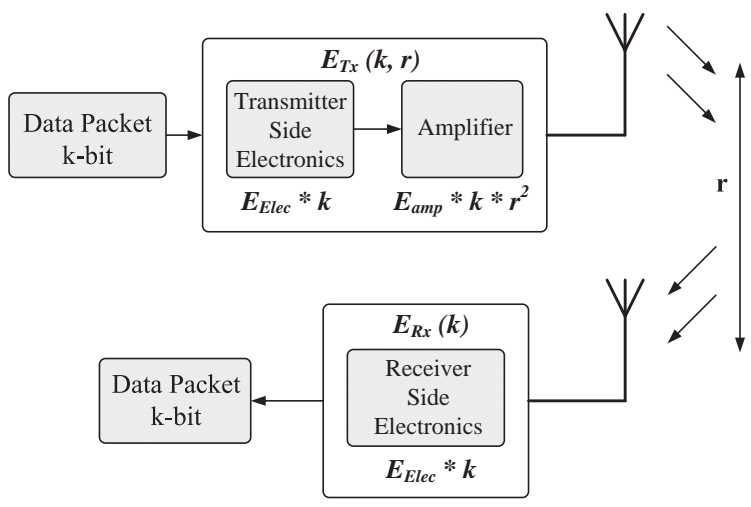

Fig. 5. Energy Consumption Model

For calculation of energy dissipation in our scheme and in [13], we consider the scenario in which two video sensors transmit total 250 packets, each of size 128 byte. $E_{\text {Elec }}$ is taken as $75 \mathrm{~nJ} / \mathrm{bit}$ and $E_{a m p}$ is taken as $100 \mathrm{pJ} / \mathrm{bit} /$ $m^{-2}$. These packets are equally disseminated between all the paths. Sensor nodes are uniformly deployed in a network of area $100 \mathrm{~m} \times 100 \mathrm{~m}$ and each sensor node has a sensing radius $r_{\text {sensing }}=40 \mathrm{~m}$. Comparison of both cases is shown in Figure 6. Our algorithm has much reduced energy consumption, which is due to the fact that we are also optimizing energy by selecting nearest next hop neighbor for path construction.

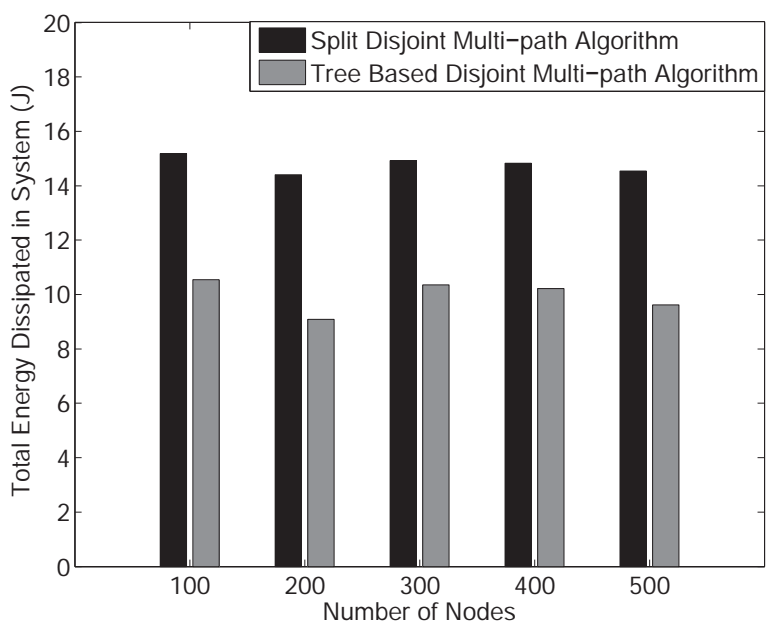

Fig. 6. Total energy consumption for transmission of 250 packets in $100 \mathrm{~m} \times 100 \mathrm{~m}$ Network for Uniform Deployment

To prove the energy efficiency of our proposed approach, we compared it with two different disjoint multipath construction 
schemes. In first algorithm [8], a simple disjoint multipath scheme is presented in which the sink is assumed to have some empirical information about its neighbor which can offer it the highest quality of data in terms of delay constrains. This node is the most preferred neighbor for the primary path construction which then selects its preferred neighbor on the basis of local information. Thus, multiple paths are constructed in this fashion. Second algorithm is discussed in [13]. We made the comparison of total energy consumption for our algorithm and the above two approaches. So, total energy dissipation for the transmission of 250 packets, each of size 128 bytes was calculated for electronics energy altering from 20 to $100 \mathrm{~nJ} /$ bit in all the cases. This comparison is shown in Figure 7, which shows that for the same end-to-end delays, our proposed algorithm is more energy efficient. This can be justified by the fact that by selecting the nearest next hop neighbor for path construction, we are performing significant energy optimization.

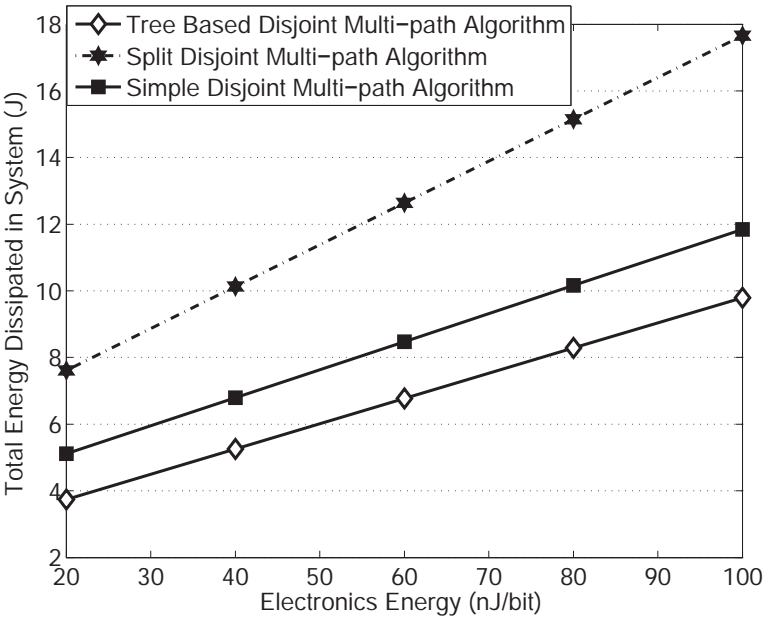

Fig. 7. Energy consumption comparison for transmission of 250 packets in $100 \mathrm{~m} \times 100 \mathrm{~m}$ Network, Nodes $=200$, for Uniform Deployment

\section{CONCLUSION}

In this paper, we demonstrate a novel multipath formation scheme for collaborative video sensor networks. In-network collaborative processing provides solution to multimedia applications with reduced energy consumption, data delivery latency and processing power. In our proposed scheme, Random trees are constructed which rapidly explore the multiple paths through the entire network. The scheme does not require geographical coordinate information of the nodes for path formation decisions. Disjoint Multiple paths of minimum energy and hop delays are constructed between the video sensor nodes themselves and between base station and video sensor nodes. Multiple paths between video sensors serve the purpose of communication between the video sensor nodes in an energy and delay efficient manner. On demand path selection priorities can be assigned for link establishment either with base station for event reporting or with other video sensors for collaborative processing according to the situation. Our algorithm surpasses the different disjoint multiple path construction schemes and simulation results show its effectiveness for collaborative video sensor networks. For future work, this scheme can be extended to include quality of service in terms of reliability of link, network lifetime and fault detection.

\section{REFERENCES}

[1] S. Khan, H. Aziz, S. Maqsood, and S. Faisal, "Clustered home area network: A beacon enabled ieee 802.15. 4 approach," in Emerging Technologies, 2008. ICET 2008. 4th International Conference on, pp. 193198, IEEE, 2008.

[2] I. Akyildiz, T. Melodia, and K. Chowdhury, "A survey on wireless multimedia sensor networks," Computer Networks, vol. 51, no. 4, pp. 921-960, 2007.

[3] I. Akyildiz, W. Su, Y. Sankarasubramaniam, and E. Cayirci, "Wireless sensor networks: a survey," Computer networks, vol. 38, no. 4, pp. 393422, 2002.

[4] J. Yick, B. Mukherjee, and D. Ghosal, "Wireless sensor network survey," Computer Networks, vol. 52, no. 12, pp. 2292-2330, 2008.

[5] Y. Gu, Y. Tian, and E. Ekici, "Real-time multimedia processing in video sensor networks," Signal Processing: Image Communication, vol. 22, no. 3, pp. 237-251, 2007.

[6] Y. Tian, J. Boangoat, E. Ekici, and F. Özgüner, "Real-time task mapping and scheduling for collaborative in-network processing in dvs-enabled wireless sensor networks," Proc. IEEE International Parallel \& Distributed Processing Symposium, pp. 1-10, Apr. 2006.

[7] Y. Tian and E. Ekici, "Cross-layer collaborative in-network processing in multihop wireless sensor networks," IEEE transactions on mobile computing, pp. 297-310, 2007.

[8] D. Ganesan, R. Govindan, S. Shenker, and D. Estrin, "Highly-resilient, energy-efficient multipath routing in wireless sensor networks," $A C M$ SIGMOBILE Mobile Computing and Communications Review, vol. 5, no. 4, pp. 11-25, 2001.

[9] L. Zhang, M. Hauswirth, L. Shu, Z. Zhou, V. Reynolds, and G. Han, "Multi-priority multi-path selection for video streaming in wireless multimedia sensor networks," Ubiquitous Intelligence and Computing, pp. 439-452, 2010.

[10] E. Felemban, C. Lee, and E. Ekici, "MMSPEED: Multipath multiSPEED protocol for QoS guarantee of reliability and timeliness in wireless sensor networks," IEEE Transactions on Mobile Computing, pp. 738-754, 2006

[11] M. Chen, V. Leung, S. Mao, and Y. Yuan, "Directional geographical routing for real-time video communications in wireless sensor networks," Computer Communications, vol. 30, no. 17, pp. 3368-3383, 2007.

[12] S. LaValle and J. Kuffner Jr, "Randomized kinodynamic planning," The International Journal of Robotics Research, vol. 20, no. 5, p. 378, 2001.

[13] S. Lee and M. Gerla, "Split multipath routing with maximally disjoint paths in ad hoc networks," in IEEE International Conference on Communications, vol. 10, pp. 3201-3205, Citeseer, 2001.

[14] L. Shu, Y. Zhang, L. Yang, Y. Wang, M. Hauswirth, and N. Xiong, "TPGF: geographic routing in wireless multimedia sensor networks," Telecommunication Systems, vol. 44, no. 1, pp. 79-95, 2010.

[15] M. Maimour, "Maximally radio-disjoint multipath routing for wireless multimedia sensor networks," in Proceedings of the 4th ACM workshop on Wireless multimedia networking and performance modeling, pp. 2631, ACM, 2008.

[16] I. Politis, M. Tsagkaropoulos, T. Dagiuklas, and S. Kotsopoulos, "Power efficient video multipath transmission over wireless multimedia sensor networks," Mobile Networks and Applications, vol. 13, no. 3, pp. 274284, 2008.

[17] W. Heinzelman, A. Chandrakasan, H. Balakrishnan, and C. MIT, "An application-specific protocol architecture for wireless microsensor networks," IEEE Transactions on wireless communications, vol. 1, no. 4, pp. 660-670, 2002 